\title{
Enhancement of light-matter interactions in slow-wave metasurfaces
}

\author{
Shiyi Xiao, ${ }^{1}$ Qiong He, ${ }^{1}$ Xueqin Huang, ${ }^{2}$ Shiwei Tang, ${ }^{1}$ and Lei Zhou ${ }^{1, *}$ \\ ${ }^{1}$ State Key Laboratory of Surface Physics and Physics Department, Fudan University, Shanghai, 200433, China \\ ${ }^{2}$ Department of Physics, The Hong Kong University of Science and Technology, Clear Water Bay, Kowloon, Hong Kong, China
}

(Received 1 October 2011; revised manuscript received 2 February 2012; published 24 February 2012)

\begin{abstract}
We show that an ultrathin metamaterial can trap photons for a long time, with the measured longest trapping time being $\sim 5 \mathrm{~ns}$ for a microwave pulse with a center frequency of $5.5 \mathrm{GHz}$. Such photon-trapping effect is governed by the anomalous dispersion and surface plasmon excitations of the system, which is more significant in thinner samples owing to stronger mode hybridizations. Light-matter interactions are remarkably enhanced inside these structures, leading to perfect omnidirectional light absorption and dramatically enhanced nonlinear generations, which are demonstrated by microwave experiments and full-wave simulations.
\end{abstract}

DOI: 10.1103/PhysRevB.85.085125

PACS number(s): 78.67.Pt, 42.25.Bs, 42.65.Ky, 78.20.-e

Light-matter interactions (LMI's) have generated lots of fascinating phenomena in condensed matter physics and optics, such as nonlinear harmonic generations, ${ }^{1}$ light absorption, ${ }^{2}$ and magneto-optical kerr effect, ${ }^{3}$ which have demonstrated many applications in practice. However, such effects are typically weak in conventional materials due to short LMI time, because light travels too fast. Available approaches to slow down light include the Bragg mechanism in photonic crystals $(\mathrm{PC})^{4-6}$ and the electromagnetically induced transparency mechanism in atomic gases. ${ }^{7,8}$ However, these approaches ${ }^{4-8}$ typically require an optically thick medium, and it is not guaranteed that incident photons can completely couple into the slow-wave structures. Metamaterials (MTM) offer many expanded freedoms to control electromagnetic (EM) wave propagations, with several fascinating phenomena already demonstrated, including negative refraction, ${ }^{9}$ subwavelength imaging, ${ }^{10}$ and invisibility cloaking. ${ }^{11}$ Recently, MTMbased slow-wave structures were proposed, including photonic systems with negative-refraction MTM components ${ }^{12-14}$ or Fano resonance-based MTM's. ${ }^{15-17}$ Still, these systems ${ }^{9-17}$ were built upon bulk slow-wave mechanisms. In addition, using such structures to enhance LMI's has not yet been explicitly demonstrated. In this paper, we demonstrate by both experiment and theory that a specific ultrathin MTM supports slow-wave propagations, which can perfectly couple to external fast waves. In contrast to conventional systems, ${ }^{4-17}$ here the physics is governed by a surface-related mechanism so that the slow-wave effect is more dramatic in thinner samples. Our metasurfaces can remarkably enhance LMI's, with two explicit examples (i.e., perfect light absorption and enhanced nonlinear generations) demonstrated by microwave experiments and self-consistent full-wave simulations.

As schematically shown in Fig. 1, our structure is a sandwich system consisting of a metal plate with a periodic array of complex slit patterns, a dielectric spacer, and a metallic substrate. The thicknesses of the top two layers are $h_{1}$ and $h_{2}$, respectively. The inset to Fig. 1 shows a picture of part of a fabricated microwave sample. We first deduced the reflection time delay $(\Delta T)$ of a microwave pulse, incident on the structure (with the spacer layer as air) and then reflected back to the receiver (see Fig. 1), from the reflection measurements. With a metallic substrate on the back, our system is always $100 \%$ reflective for EM waves. In our experiment, we used a vector network analyzer (Agilent 8722ES) to measure the reflection spectrum $r(\omega) \equiv e^{i \phi(\omega)}$ for transverse-magnetic (TM) polarized plane waves (with $\vec{H} \| \hat{y}$ ) at an incident angle $\theta=20^{\circ} .^{18}$ With $r(\omega)$ obtained, we then constructed a Gaussian pulse with center frequency $\omega$ using the formula $F(z, t)=$ $\int e^{-\left(\omega^{\prime}-\omega\right)^{2} / 2 \Delta^{2}}\left[e^{-i \omega^{\prime} z / c}+r\left(\omega^{\prime}\right) e^{i \omega^{\prime} z / c}\right] e^{i \omega^{\prime} t} d \omega^{\prime}$ and calculated the time delay $(\Delta T)$ by monitoring the peak-position motion of the pulse (see Supplemental Material). ${ }^{19}$ The time that the pulse travels in air is deduced so that $\Delta T$ precisely measures the net time in which the pulse is trapped inside the structure. The $\Delta T(\omega)$ spectrum deduced from experimental data is depicted in Fig. 2(a), which is in excellent agreement with the simulation results based on a finite element method (FEM) ${ }^{20}$ We note that while $\Delta T \approx 0$ at most frequencies, $\Delta T$ significantly increases in a frequency window centered at $\sim 5.5 \mathrm{GHz}$ (corresponding to a wavelength $\lambda \approx 55 \mathrm{~mm}$ ), indicating that our structure can trap the EM pulse for a long time. To understand the noted phenomena, we performed FEM simulations $^{20}$ at three typical frequencies. Insets to Fig. 2(a) show that while EM waves are directly reflected back by the upper surface at both $4 \mathrm{GHz}$ and $7 \mathrm{GHz}$, they could penetrate inside the apertures at $5.5 \mathrm{GHz}$ and stay there for a long time ( $\sim 2 \mathrm{~ns}$ ) before leaving the structure (see Supplemental Material) ${ }^{19}$ Recalling that the total thickness of our system is only $h=h_{1}+h_{1}=4 \mathrm{~mm}$, we found that the "effective" wave speed inside our structure is $v_{g}=2 h / \Delta T \sim c / 100$ at $\sim 5.5 \mathrm{GHz}$. The slow-wave effect is robust against the polarization and incident angle of the input beam, and similar spectra were obtained for transverse-electric (TE) polarization cases with different incident angles.

Figure 2(b) depicts how $\Delta T$ depends on two parameters, $h_{1}$ and $h_{2}$, obtained by FEM simulations. In the left corner where both $h_{1}$ and $h_{2}$ are large, $\Delta T$ depends linearly on $h_{1}$ and $h_{2}$, suggesting the dominance of a bulk effect. Intriguingly, as $h_{1}, h_{2} \rightarrow 0,{ }^{21} \Delta T$ does not decrease but rather increases dramatically. To gain a clearer picture of such an anomalous effect, we show by solid and dotted lines in Fig. 3(a) how the calculated $\Delta T$ depends on $h_{1}$ with $h_{2}$ fixed at two distinct values. The common linear dependences exhibited by the two curves in the region $h_{1}>10 \mathrm{~mm}$ suggest an analytical formula for $\Delta T$ :

$$
\Delta T \approx \Delta T_{s}+2 h_{1} / v_{z}
$$




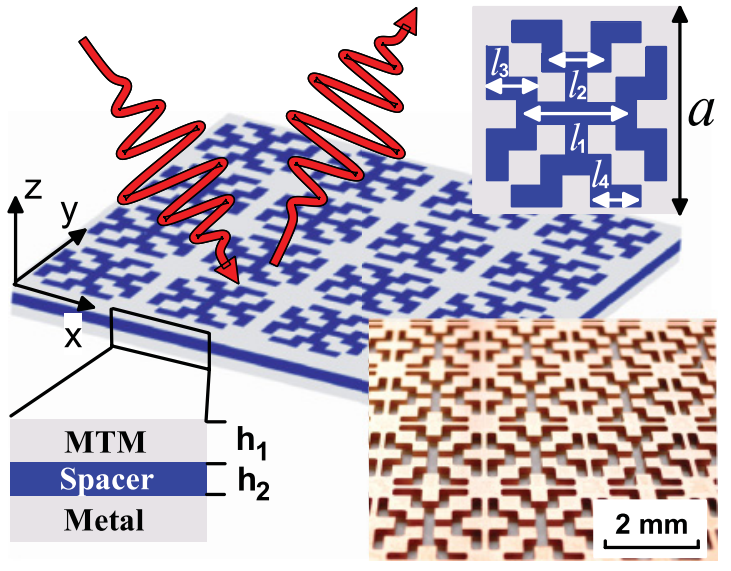

FIG. 1. (Color online) Geometry of the designed metasurface and picture of part of a fabricated sample with $a=20 \mathrm{~mm}, w=2 \mathrm{~mm}$, $l_{1}=10 \mathrm{~mm}, l_{2}=5 \mathrm{~mm}, l_{3}=5 \mathrm{~mm}, l_{4}=5 \mathrm{~mm}, h_{1}=2 \mathrm{~mm}$, and $h_{2}=$ $2 \mathrm{~mm}$. The metallic material used here is copper, and the spacer is just an air gap.

We note that each aperture in the upper metallic plate forms a waveguide as $h_{1}$ becomes large (see Fig. 1), and therefore, $v_{z}$ in Eq. (1) precisely represents the group velocity for photons traveling inside such a waveguide. On the other hand, the term $\Delta T_{s}$, which does not depend on $h_{1}$, must be a surface-related remaining contribution. Figure 3(a) already implies that $\Delta T_{s}$ strongly depends on $h_{2}$. The FEM-calculated $\Delta T \sim h_{2}$ curve (with $h_{1}$ fixed at $1 \mathrm{~mm}$ ), depicted in Fig. 3(b), verifies that $\Delta T_{s}$ inversely depends on $h_{2}$ and increases dramatically as $h_{2} \rightarrow 0 .{ }^{21}$ Microwave experiments on a series of samples with different $h_{1}$ and $h_{2}$ verified such anomalous behaviors of $\Delta T_{s}$ in small- $h$ regions, as shown by the open circles in Figs. 3(a) and 3(b). These singular thickness dependences suggest that $\Delta T_{s}$ must come from a surface-related mechanism.

To explore the physics of such an anomalous effect, we depicted in Fig. 3(c) the numerically computed dispersion curves for the propagating modes inside the aperture waveguides (assuming an infinite $h_{1}$ ) and the spoof surface plasmon polaritons (SPP) on the structural surface, respectively. As shown in Fig. 3(c), the shape resonance of the aperture results in a cutoff for the waveguide mode at $f_{0} \sim 4.4 \mathrm{GHz}$ so that the (a)

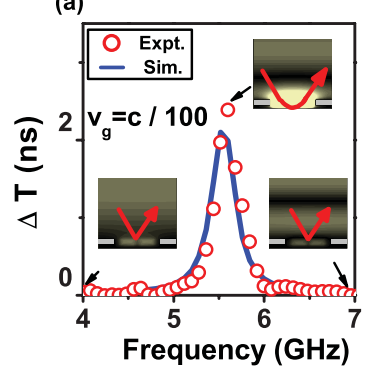

(b)

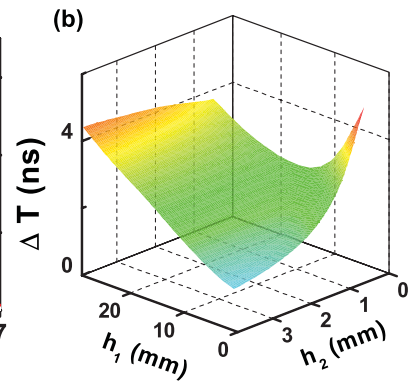

FIG. 2. (Color online) (a) Measured (red dot) and simulated (blue lines) delay time $(\Delta T)$ spectra for the fabricated metasurface. Insets show the calculated electric-field distributions under external radiations at different frequencies. (b) FEM-simulated delay time $\Delta T$ (calculated at frequency $5.5 \mathrm{GHz}$ ) as a function of $h_{1}$ and $h_{2}$ with other geometrical parameters the same as those in Fig. 1.

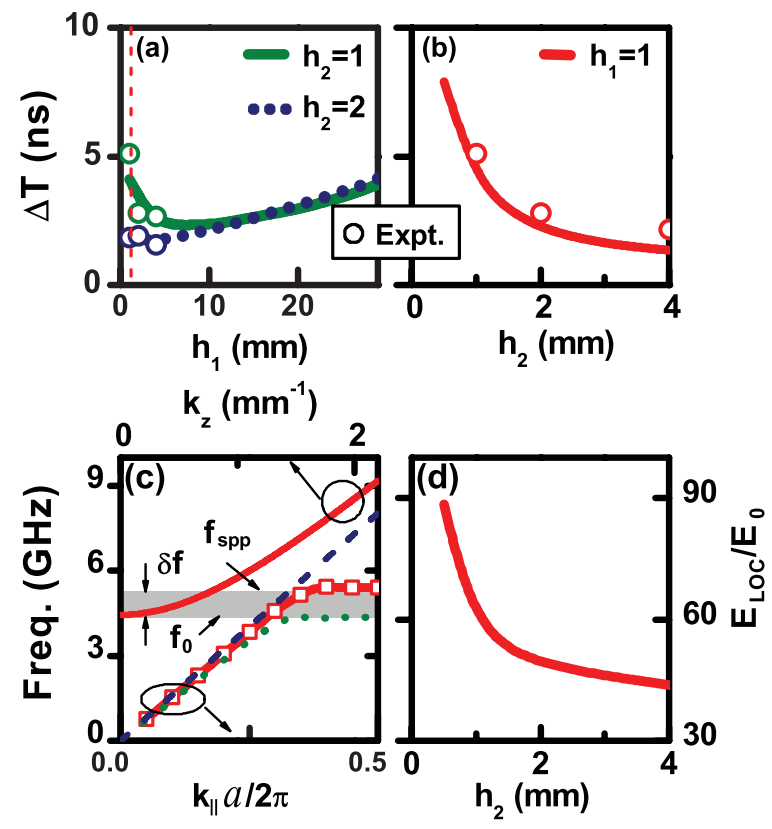

FIG. 3. (Color online) (a) Simulated (lines) and measured (circles) $\Delta T$ as functions of $h_{1}$ (thickness of the upper metallic layer) with $h_{2}$ (thickness of the spacer layer) fixed as $1 \mathrm{~mm}$ and $2 \mathrm{~mm}$, respectively. (b) Simulated (lines) and measured (circles) $\Delta T$ as functions of $h_{2}$ with $h_{1}$ fixed as $1 \mathrm{~mm}$ [corresponding to the red dashed line in Fig. 3(a)]. Other geometrical parameters of the systems studied in (a) and (b) are kept the same as those in Fig. 1. (c) Calculated dispersion curves for the propagating mode inside the aperture waveguide (solid line) and the spoof SPP on a stand-alone upper metallic plate (green dotted line) and on the whole sandwich structure (squares) for the fabricated sample. The dashed line denotes the light line. (d) FEM-computed local-field enhancement at the hot spot inside the device as a function of $h_{2}$ with $h_{1}=1 \mathrm{~mm}$.

bulk mode group velocity is $v_{z} \sim \partial \omega / \partial k_{z} \rightarrow 0$ at frequencies near the cutoff. Meanwhile, it was demonstrated that a metallic plate with periodic array of apertures (i.e., the upper metallic plate) was a plasmonic MTM to support spoof SPP with plasmon frequency right at the waveguide cutoff [see the green dotted line in Fig. 3(c)].$^{22,23}$ However, in the present sandwich structure with an additional metallic substrate, strong interactions between the MTM and its image (though the substrate) result in a branch of antisymmetrical hybridized SPP. As shown in Fig. 3(c), we found that the plasmon frequency of such an SPP branch is blueshifted $\left(f_{\mathrm{SPP}}=5.5\right.$ $\mathrm{GHz}$ ) as compared to $f_{0}$, caused by the hybridization effect. The frequency splitting $\delta f=f_{\mathrm{SPP}}-f_{0}$ is more significant as $h_{1}, h_{2} \rightarrow 0$ because of the enhanced mode hybridization effect.

Figure 3(c) established a clear physical picture for the observed photon-trapping effect. The incoming EM wave first excites the spoof SPP, which propagate very slowly on the structural surface. It costs a very long time $\left(\sim \Delta T_{S}\right)$ for the SPP wave to penetrate into the waveguides because each aperture has a very small opening. ${ }^{24}$ Wave propagation inside the waveguide is again very slow because $v_{z} \ll c$ here, resulting in an additional delay time $\sim h_{1} / v_{z}$. For the system with the small $h_{1}, h_{2},{ }^{21} \Delta T$ is mainly contributed by the surface term $\Delta T_{s}$. We found that stronger mode hybridization in the 
smaller $h_{2}$ case results in stronger local field enhancement, as shown in Fig. 3(d). Similar hybridization effects were found in other coupled systems..$^{25,26}$ The stronger the hybridization is, the longer the surface delay time $\Delta T_{S}$ is to establish such a hybridized SPP state. This explains the singular dependence of the slow-wave effect on $h_{1}, h_{2}$, as observed in our structure [see Fig. 2(b)].

Intuitively, one may view our metasurface as a special cavity with the $Q$-factor dictated by the coupling between the hybridized SPP and external radiations. However, the mechanism here is quite different from that in a conventional Fabry-Pérot (FP) cavity, because we do not have multiple scatterings of light inside the system and the working frequency is nearly independent on the device thickness. In addition, our system possesses a much smaller mode volume $(V)$ than a conventional FP cavity whose thickness is comparable to wavelength. As a result, our system has a larger Purcell factor $(\sim Q / V)^{27}$ than the FP one, leading to better performances on enhancing the LMI's.

Consider a beam of light with a photon density $n_{0}$ incident on our metasurface. In the stabilized configuration, the conservation of photons yields $n_{0} \cdot c \cdot A=n_{\mathrm{sw}} \cdot v_{g} \cdot S_{a}$, where $n_{\mathrm{sw}}$ is the photon density inside the slow-wave medium, $S_{a}$ denotes the area of the aperture, and $A=a^{2}$ is the unit cell area. Therefore, we get

$$
n_{\mathrm{sw}}=n_{0} \cdot\left(c / v_{g}\right) \cdot\left(A / S_{a}\right) .
$$

Equation (2) shows that photon density is significantly enhanced inside our structure by two factors: the slow-down of light speed to compress the wave longitudinally $\left(c / v_{g} \gg\right.$ 1) and the aperture effect to squeeze the wave laterally $\left(A / S_{a} \gg 1\right)$. Inserting materials with particular light-matter responses into the apertures, we expect the LMI's to be significantly enhanced due to the enhanced local photon density, especially for those LMI phenomena based on multiphoton processes. In what follows, we took light-absorption and third-harmonic generation (THG) as two explicit examples to verify our predictions.

Recently, absorbing light with $100 \%$ efficiency attracted much interest. Several approaches have been proposed based on different mechanisms such as impedance matching, ${ }^{28}$ gradient-index antireflection, ${ }^{29}$ and plasmonic resonance. ${ }^{30}$ Here, we show that a perfect absorber can be made based on our metasurface. Inserting low-absorptive FR4-PCB powders (with $\varepsilon=1.5+0.03 i$ ) into the apertures and replacing the air gap by a 2-mm-thick low-absorptive dielectric spacer (with $\varepsilon=3.9+0.075 i$ ), we performed microwave experiments to measure the absorption spectra of the entire system. Figure 4(a) shows the absorption spectra, $A=1-R-T,{ }^{31}$ measured with input waves taking TE or TM polarizations at an incident angle $\theta=15^{\circ}$. Absorbance is significantly enhanced in a frequency window centered at $\sim 4 \mathrm{GHz}$ with peak absorption $\sim 100 \%{ }^{32}$ The fact that the absorbance can reach $100 \%$ reinforced our previous notion that photons can perfectly couple into our device, because absorption only happens inside the device. In addition, the long trapping time ensures that the photons are efficiently absorbed before they leave the device. This is quite remarkable because the total thickness of our structure is only $\sim \lambda / 20$ and can in principle be further reduced

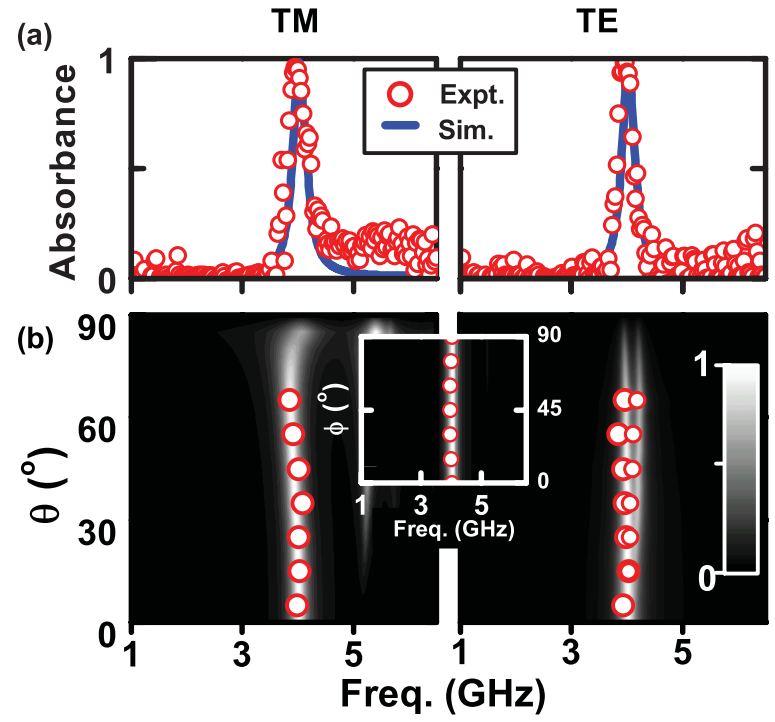

FIG. 4. (Color online) (a) Measured (circles) and simulated (lines) EM wave absorption spectra under radiations with TM or TE polarizations and $\theta=15^{\circ}, \phi=0^{\circ}$. (b) FEM-computed absorbance versus incidence angle and frequency under radiations with TM or TE polarizations and $\phi=0^{\circ}$; circles denote the absorption peak position measured experimentally. Inset shows the measured (circles) and simulated $\phi$-dependence of the absorption peak (setting $\theta=15^{\circ}$ ).

according to Figs. 2 and 3. Simulation results (lines) $)^{20}$ are in excellent agreement with the experiment, both showing that the perfect absorption effect is robust against the polarization, incident angle $\theta$, and azimuthal angle $\phi^{18}$ of the in-plane $\mathbf{E}$ vector [see Fig. 4(b)]. The incidence-angle robustness can be understood from Fig. 3(c), where the SPP band is found very flat, and thus the slow-wave peak must be insensitive to the incidence angle.

We designed a metasurface working at the terahertz regime and employed finite-difference-time-domain (FDTD) simulations ${ }^{33}$ to demonstrate the THG enhancement effect, assuming that all apertures were filled with nonlinear material with permittivity $\varepsilon_{r}=2.56+\chi^{(3)}|\vec{E}|^{2}{ }^{34}$ In our simulations, we assumed that the device is made by $\mathrm{Au}$, described as a lossy metal with conductivity $\sigma=4.09 \times 10^{7} \mathrm{~S} / \mathrm{cm} .{ }^{35}$ The calculated THG spectrum (not shown) of the entire system exhibits a pronounced peak at $0.3 \mathrm{THz}$, in consistency with the time-delay spectrum of our device calculated with nonlinearity removed. To quantify the THG enhancement achieved by our device, we depicted in Fig. 5(a) the power of THG $I_{3 \omega}$ versus that of the incoming light $I_{0}$, calculated at the working frequency $0.3 \mathrm{THz}$ (corresponding to $\lambda=1 \mathrm{~mm}$ ). As a reference, we showed in the same figure the calculated $I_{3 \omega} \sim I_{0}$ curve for a slab formed by nonlinear materials with the same volume. We found $I_{3 \omega} \propto I_{0}^{3}$ as expected in both cases, but our structure has enhanced the THG power by more than $10^{4}$ times! Although a recent experiment has demonstrated that THG can also be enhanced inside a slow-wave PC waveguide, ${ }^{36}$ the advantage of our structure is that the device can be much thinner than wavelength. Finally, we note that the slow-wave effect is sensitive to the losses of the metal 

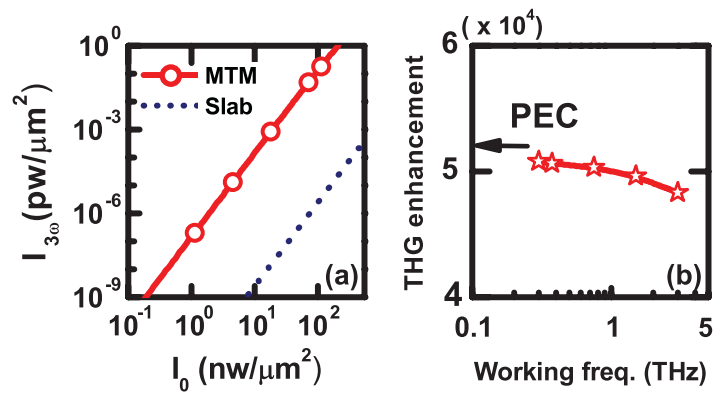

FIG. 5. (Color online) (a) FDTD-calculated output power of THG $\left(I_{3 \omega}\right)$ versus that of incoming light $\left(I_{0}\right)$ at the operation frequency $0.3 \mathrm{THz}$ for the slow-wave device (with $a=260 \mu \mathrm{m}, w=$ $h_{1}=h_{2}=26 \mu \mathrm{m}$, and $l_{1}=2 l_{2}=2 l_{3}=2 l_{4}=130 \mu \mathrm{m}$ ) filled with nonlinear materials (solid line) and a slab formed by nonlinear materials of the same volume (dashed line). (b) Calculated THG enhancement achieved by slow-wave devices working at different frequencies, with arrow denoting the value achieved by a slow-wave device made by perfect electric conductors (PEC). forming the device. As shown in Fig. 5(b), we scaled the device's size to change the working frequency from 0.3 to $3 \mathrm{THz}$ and found through FDTD simulations that the THG enhancement gradually decreases as the working frequency increases. Obviously, the enhanced metallic losses diminish the slow-wave effect as frequency increases.

In conclusion, we demonstrate by both experiments and simulations that a particular type of metasurface can trap photons for a long time, with physics governed by anomalous dispersion and surface plasmon excitations of the system. LMI's are remarkably enhanced in such devices, leading to perfect light absorption and enhanced nonlinear harmonic generations, which are demonstrated by microwave experiments and full-wave simulations.

We thank Y. R. Shen and C. T. Chan for discussions. This work was supported by NSFC (Nos. 60990321, 11174055) and MOE of China (No. B06011). XQH was supported by Hong Kong RGC Grant No. 600209. *phzhou@fudan.edu.cn

${ }^{1}$ Y. R. Shen, The Principles of Nonlinear Optics (Wiley, New York, 1973).

${ }^{2}$ H. A. Atwater and A. Polman, Nat. Mater. 9, 205 (2010).

${ }^{3}$ G. P. Zhang, W. Hübner, Georgios Lefkidis, Yihua Bai, and Thomas F. George, Nat. Phys. 5, 499 (2009).

${ }^{4}$ M. Notomi, K. Yamada, A. Shinya, J. Takahashi, C. Takahashi, and I. Yokohama, Phys. Rev. Lett. 87, 253902 (2001).

${ }^{5}$ X. Letartre, C. Seassal, C. Grillet, P. Rojo-Romeo, P. Viktorovitch, M. Le Vassor d'Yerville, D. Cassagne, and C. Jouanin, Appl. Phys. Lett. 79, 2312 (2001).

${ }^{6}$ Toshihiko Baba, Nat. Photonics 2, 465 (2008).

${ }^{7}$ L. V. Hau, S. E. Harris, Z. Dutton, and C. H. Behroozi, Nature (London) 397, 594 (1999).

${ }^{8}$ A. Kasapi, M. Jain, G. Y. Yin, and S. E. Harris, Phys. Rev. Lett. 74, 2447 (1995).

${ }^{9}$ R. A. Shelby, D. R. Smith, and S. Schultz, Science 292, 77 (2001).

${ }^{10}$ N. Fang, H. Lee, C. Sun, and X. Zhang, Science 308, 534 (2005).

${ }^{11}$ D. Schurig, J. J. Mock, B. J. Justice, S. A. Cummer, J. B. Pendry, A. F. Starr, and D. R. Smith, Science 314, 977 (2006).

${ }^{12}$ K. L. Tsakmakidis, A. D. Boardman, and O. Hess, Nature (London) 450, 397 (2007).

${ }^{13} \mathrm{~J}$. He and S. He, IEEE Microw. Wireless Compon. Lett. 16, 96 (2005).

${ }^{14}$ T. Jiang, J. M. Zhao, and Y. J. Feng, Opt. Express 17, 170 (2009).

${ }^{15}$ N. Papasimakis, V. A. Fedotov, N. I. Zheludev, and S. L. Prosvirnin, Phys. Rev. Lett. 101, 253903 (2008).

${ }^{16}$ S. Zhang, D. A. Genov, Y. Wang, M. Liu, and X. Zhang, Phys. Rev. Lett. 101, 047401 (2008).

${ }^{17}$ C. Wu, A. B. Khanikaev, and G. Shvets, Phys. Rev. Lett. 106, 107403 (2011).
${ }^{18}$ Here, $\theta$ defines the angle between the $z$ axis and the incident $\mathbf{k}$ vector, and $\varphi$ defines the angle between the $x$ axis and the $\mathbf{k}$ vector projected on the xy plane.

${ }^{19}$ See Supplemental Material at http://link.aps.org/supplemental/ 10.1103/PhysRevB.85.085125 for the deduction process and the simulations on pulse movements.

${ }^{20}$ COMSOL Multiphysics 3.5, developed by COMSOL Inc (Burlington, USA, 2008)

${ }^{21} h_{1}$ should be larger than the skin depth of metal, which is $\sim 1 \mu \mathrm{m}$ in $3 \mathrm{GHz} ; h_{2}$ should be larger than several tens of nanometers to prevent quantum tunneling of electrons.

${ }^{22}$ J. B. Pendry, L. Martín-Moreno, and F. J. Garcia-Vidal, Science 305, 847 (2004).

${ }^{23}$ Xueqin Huang, Shiyi Xiao, Dexin Ye, Jiangtao Huangfu, Zhiyu Wang, Lixin Ran, and Lei Zhou, Opt. Express 18, 10377 (2010).

${ }^{24}$ The spoof SPP also plays a crucial role of coupling external radiations perfectly through the upper metallic plate, because of the extraordinary optical transmission mechanism.

${ }^{25}$ R. F. Oulton, V. J. Sorger, T. Zentgraf, R. M. Ma, C. Gladden, L. Dai, G. Bartal, and X. Zhang, Nature (London) 461, 629 (2009).

${ }^{26}$ S. H. Mousavi, A. B. Khanikaev, B. Neuner, Y. Avitzour, D. Korobkin, G. Ferro, and G. Shvets, Phys. Rev. Lett. 105, 176803 (2010).

${ }^{27}$ T. J. Kippenberg, A. L. Tchebotareva, J. Kalkman, A. Polman, and K. J. Vahala, Phys. Rev. Lett. 103, 027406 (2009)

${ }^{28}$ N. I. Landy, S. Sajuyigbe, J. J. Mock, D. R. Smith, and W. J. Padilla, Phys. Rev. Lett. 100, 207402 (2008).

${ }^{29}$ T. V. Teperik, F. J. García de Abajo, A. G. Borisov, M. Abdelsalam, P. N. Bartlett, Y. Sugawara, and J. J. Baumberg, Nat. Photonics 2, 299 (2008).

${ }^{30}$ E. Rephaelia and S. Fan, Appl. Phys. Lett. 92, 211107 (2008).

${ }^{31}$ Thanks to the metallic plate on the back, here we have $T \equiv 0$.

${ }^{32}$ The absorption peak is redshifted with respect to the slow-wave peak as shown in Fig. 2(a), caused by the filling dielectric medium and the dielectric spacer. 
${ }^{33}$ EastFDTD v2.0 Beta, DONGJUN Science and Technology Co., China.

${ }^{34}$ We set $\chi^{(3)}=2 \times 10^{6} \mathrm{erg} / \mathrm{cm}^{3}$ in FDTD simulations, but the THG enhancement does not depend on the absolute value of $\chi^{(3)}$. Nonlinear responses were considered self-consistently by iterating $\varepsilon_{r}$ with the local E-field value at each time step in the simulations.
${ }^{35}$ H. Tao, C. M. Bingham, A. C. Strikwerda, D. Pilon, D. Shrekenhamer, N. I. Landy, K. Fan, X. Zhang, W. J. Padilla, and R. D. Averitt, Phys. Rev. B 78, 241103 (2008).

${ }^{36}$ B. Corcoran, C. Monat, C. Grillet, D. J. Moss, B. J. Eggleton, T. P. White, L. O'Faolain, and T. F. Krauss, Nat. Photonics 3, 206 (2009). 